\title{
Composite Simulation as Example of Industry Experience
}

\author{
Joachim Bauer \\ Director Sales Central Europe - DELMIA \\ Dassault Systemes Deutschland GmbH \\ joachim.bauer@3ds.com
}

\begin{abstract}
Development of composite parts is characterized by a high degree of parallel processes and dependencies between structural analysis, design and manufacturing planning. Composites allow a higher degree of freedom in the design of products than other materials. On the other hand manufacturing processes for composites influence material properties, product characteristics and quality decisively. Increasing requirements in terms of product reliability, low production costs and development time require software solutions, which allow to design and virtually test the behavior of composite parts and their manufacturing processes. Software applications for composite parts allow the realistic simulation of the product in the full life cycle, from the manufacturing through the operation to the recycling processes. But despite the consequent usage of simulation applications ramping up a real production of complex composite products often provides some surprises and new experiences. Intelligent PLM software solutions support here to explore unforeseen dependencies between influencing parameters and allow to optimize the real production in regards of quality and manufacturing costs. Systematic collection of manufacturing data and automatic pattern recognition are the key for reliable and reproducible manufacturing processes.
\end{abstract}

Keywords: 3DEXPEREIENCE, 3DMaster, CATIA, DELMIA, Composites.

\section{$1 \quad$ Introduction}

The concept of 3DEXPERIENCES in the manufacturing domain is of significant importance for many companies. New production technologies, increasing product complexity, shorter product life cycles, higher product individualization and the ongoing need to reduce development and manufacturing costs are drivers for digital manufacturing solutions. Physical prototypes, trial and error approaches in the development of new manufacturing processes and unforeseen surprises with negative impacts during production ramp-up are not affordable in highly competitive markets. Production planning and manufacturing engineering based on reliable methods and simulation tools are of substantial importance for industries which manufacture goods. Dassault Systemes is an acknowledged leader in the digital manufacturing domain. With 3DEXPERIENCE the virtual modelling and simulation of real production 
environments are becoming more realistic and allow the production planner to work in a $3 \mathrm{D}$ environment with all relevant manufacturing information.

Composite Materials are found in more and more industrial applications. Due to the high potential for mass reductions companies in the aerospace and defence industry have traditionally the highest demand, followed by fast growing appliances in the transportation and mobility industry. But also consumer goods - esp. sports equipment - and other industries have requirements, which are best fulfilled by carbon or glass fibre reinforced plastics.

3DEXPERIENCE in the domain of composite products is an excellent example to demonstrate, that industrial manufacturing processes and the resulting product behaviour can be simulated and complex dependencies can be managed with high accuracy.

\section{Developing Composite Material Products}

Composite materials are characterized by very special material behaviour and production requirements. Product properties depend on many factors, which show often complex dependencies. Important material criteria like strength and stiffness are by principle non-homogenous and orthotropic and are highly dependent on the complete production process of a specific part. Material failure behaviour is very different from metals. Structural parts (e.g. for aircrafts, auto, wind turbine blades) are build in layered structures, where each layer can have different angles, thickness or even fibre material. The curing of the impregnated material is a key process. Duration, temperature and pressure define the characteristic of the cured resin in the part and the resin/fibre interaction and as a consequence the structural behaviour of the part. Therefore it is very important, that the manufacturing concept is already secured in the design phase, e.g. for static and dynamic stress analysis.
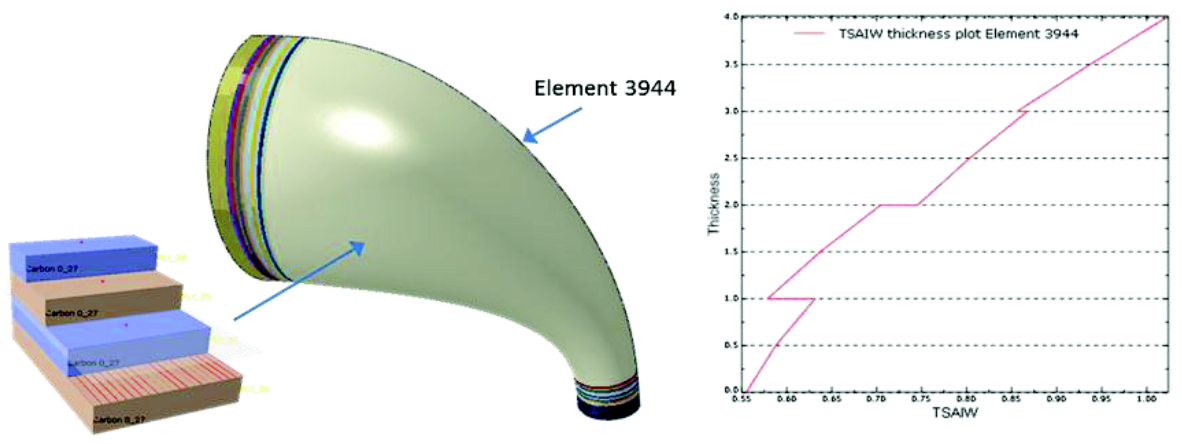

Fig. 1. Model of composite part with four layer stack-up and stress result for Finite Element

For each layer of a composite structure, the number of layers, thickness, weave, fibre angles and material have to be defined. In large structures the number of plies can vary a lot, e.g. from app. 50 layers at the root of a rotor blade to a handful at the tip. The distribution of the different layer stack ups over the part has to fulfil requirements 
on stiffness and strength, but also manufacturing boundary conditions. Size of the cut pieces are defined by production methodologies and available materials, size and position of required overlaps by structural requirements.

When a new composite part is coming into real production, quality assurance has a high priority. Many parameters have an influence and need to be considered in the case of defects. Ramping-up a serial production with secured processes to achieve a reproducible quality and fulfil cost targets is a permanent challenge.

\section{Digital Industry Experiences for Composite Simulation}

The complexity of the design and manufacturing of composite parts and the high degree of interdependencies between structural, design and manufacturing aspects generate the need for sophisticated simulations using a wide scope of methods.

One key simulation domain is the structural analysis using structure models with specialized finite elements, which allow the simulation of the behaviour of each single layer at any given location of the product. Failure of matrix or fibre material can be predicted as well as the deformation behaviour of the product under structural or thermal loads (Picture 1). The structural model can be directly derived from the design model with each layer modelled with its correct 3D representation and including all relevant material information attached as attributes. CATIA provides the necessary functionality and sufficient capacity to handle a wide range of applications. In multiple iterations between the 3DMaster design and the structural analysis based on finite elements the laminate can be optimized, in most applications to minimize weight within specified deformation and strength envelopes. The close link between SIMULIA as simulation system and CATIA as lead application for the 3DMaster facilitates fast iterations reducing the risk of data loss.

Manufacturing simulation has also its starting point at the 3DMaster model. The clarification of the question, if the selected fabrics can be draped as required for the part shape is often at the beginning. Weave, thickness, size and form of the cut pieces as well as the way they are applied into the mould are parameters which are taken into account in the process of drape simulation (Picture 2). The deformation of the cut fabric when applied into the mould and resulting fibre angle deviations can be calculated and visualized. Different lay-up procedures can be evaluated against each other and it can be verified, if the part can be manufactured with the selected material and how the fabric needs to be cut. As required, the 3D model can be adapted (e.g. by choosing a different fabric weight or weave) and more detailed e.g. by reflecting the cut pieces as defined by manufacturing engineering. Of course direct structural analysis verification can be executed for changes which might have an impact on the part behaviour. Of course it is always possible to track that part mass stays below the aloud limit.

With the shape and laminate definition accomplished, manufacturing planning and simulation can enter in a more detailed phase using the available 3D data. The documentation of the laminate stack-up can be used to generate work instructions for manual operations and a process plan. 


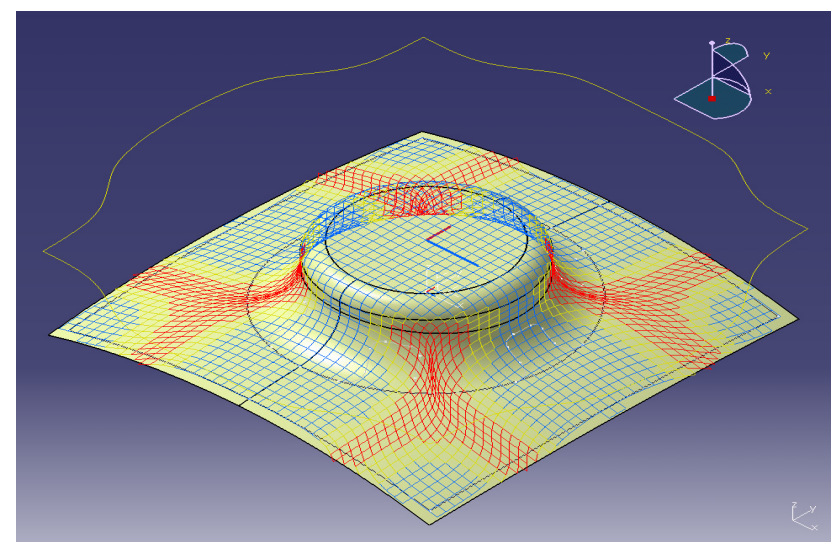

Fig. 2. Drape simulation showing fibre angle deflections of a bi-directional fabric

Applying STM (Standard time measurement) methods and linking the process plan with resource and layout information delivers important information for work load balancing and manufacturing costs calculation. This is the entry point into work cell simulation and plant design, including the possibility of life like ergonomic simulation of worker operations. DELMIA as Dassault Systemes Digital Manufacturing brand provides a wide scope of applications for realistic simulation experiences addressing these domains.

More and more automated manufacturing processes are used to manufacture composite products. Filament winding, tape laying, RTM of preformed fabrics and automated work cells with handling robots and hydraulic presses are used for many applications. Aerospace is the industry with the biggest share of these manufacturing methods, but they have also found entrance in other industries. Machine simulation and off-line programming is providing high advantages for machine builders as well as for companies, who use automated processes to build composite parts. A realistic simulation combined with the capability to download machine programs reduces downtimes and manufacturing risks.

All these applications are used to support the product development and manufacturing engineering of composite parts and are applied prior to the real production. Not all aspects in composite manufacturing can be simulated and by principle the real world is not always compliant with the assumptions in the virtual models. The high degree of dependencies between the quality of composite parts and manufacturing and environmental parameters requires add. tools. Often more than hundred parameters need to be tracked and interdependencies to be identified, if quality issues appear despite the fact, that all parameters are in their allowed range.

DELMIA Operations Intelligence provides the intelligence to identify hidden pattern and helps manufacturing experts to eliminate issues in the real production to minimize rework and scrap. 


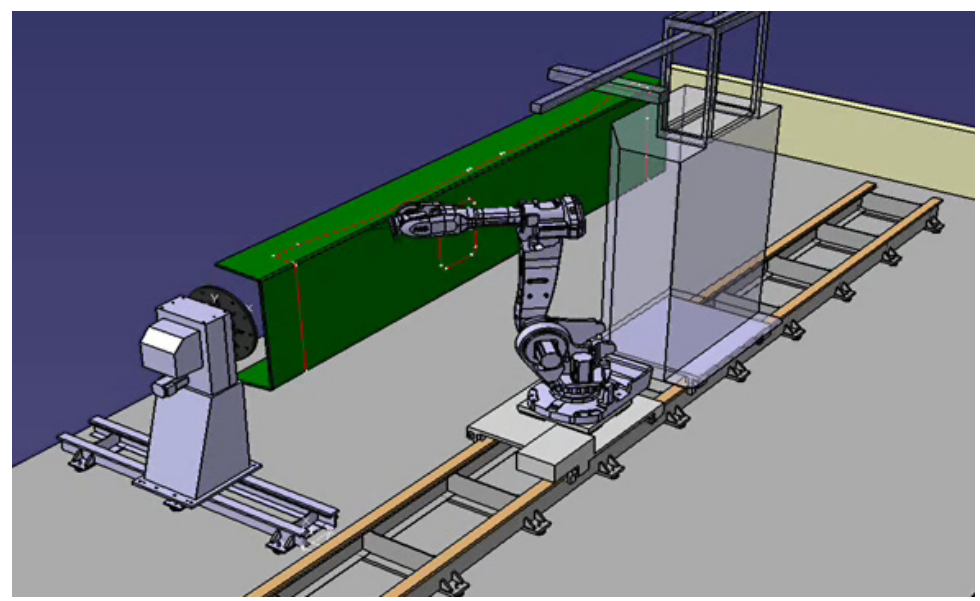

Fig. 3. Model of an automated tape laying workcell

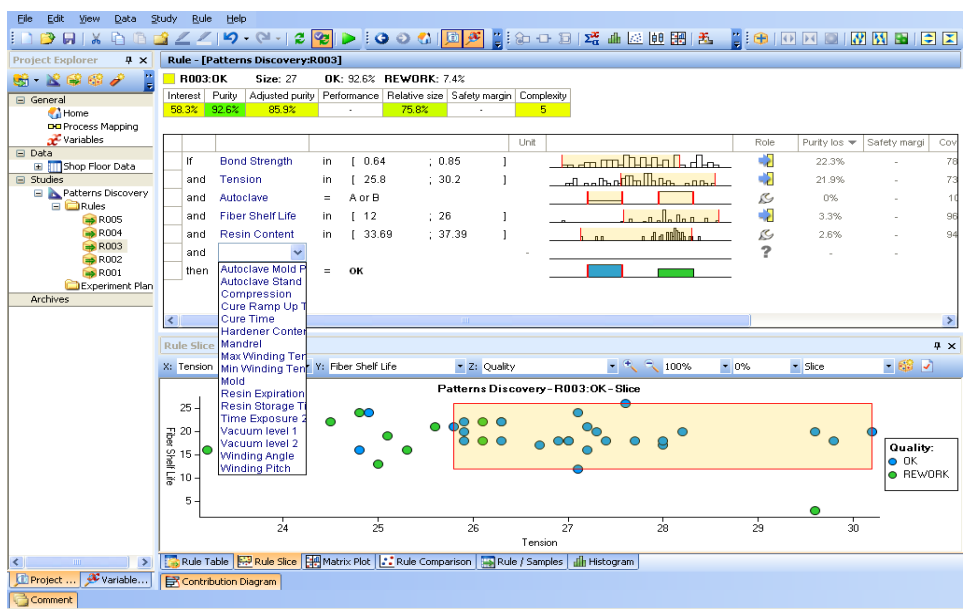

Fig. 4. Visualize and manage identified rules for composite manufacturing processes

\section{Conclusions}

The complexity of composite products requires powerful modelling and simulation applications. For the efficient usage in an industrial environment a high degree of integration between design, structural simulation and manufacturing engineering tools is necessary. A detailed 3DMaster model with all information relevant for product and manufacturing simulation is the core element. Simulation applications with direct access to this information can provide virtual experiences which reduce significantly risks and costs when developing and manufacturing products and help to avoid bad experiences in the real world. 